\title{
Using Intimacy, Chronology and Zooming to Visualize Rhythms in Email Experience
}

\author{
Mirko Mandic, Andruid Kerne \\ Interface Ecology Lab | Center for Study of Digital Libraries | Computer Science Department \\ Texas A\&M University, College Station, TX, 77843, USA \\ \{mirkokrug, andruid\}@csdl.tamu.edu
}

\begin{abstract}
Experiences of intimacy and connectedness through social networks are vital to human sense of well-being. We live in an electronic habitat. Electronic mail functions as a medium of interpersonal exchange. As it accumulates, email data becomes more than a collection of reminders. It is a diary we didn't know we were keeping, and a potential source of valuable insight into the structure and dynamics of one's social network. Current interfaces do little to help users see patterns of social interaction within email data.

We introduce a multiscale email interface that utilizes computed intimacy measures and chronology as parameters for information visualization. Rhythms of intimacy in email experience are made visible as patterns of color and shapes in a zoomable chronological grid. Qualitative user experience data indicates that such an email visualization can provide striking insights into the experience of social connectedness over time. These insights potentially enable users to better manage how they invest time and energy into personal and work relationships, and thus to improve overall sense of well-being.
\end{abstract}

\section{Author Keywords}

Email, email visualization, email interface, intimacy, intimate computing, experience design, social networks.

\section{ACM Classification Keywords}

H.4.3 Communications Applications: Electronic Mail.

\section{INTRODUCTION}

Email has developed into one of the most extensively used computer applications and a ubiquitous communication medium. As of four years ago, there were about a billion email accounts worldwide [3] and in 1998, an estimated 3 billion messages were sent daily only in the USA [4]. Email has become our primary electronic "habitat" [1]. However, beyond a few notable exceptions $[1,4,9,11,12]$ the form of

Copyright is held by the author/owner(s)

CHI 2005, April 2-7, 2005, Portland, Oregon, USA.

ACM 1-59593-002-7/05/0004. the medium hasn't developed in a manner that matches its rise in significance, and email interfaces have gone through very few changes since their inception. Popular email applications enable users to organize messages in folders. This is problematic, and as a consequence, about half of email users do not sort their messages into folders, but instead, keep all of them in their inboxes [13].

Email is a primary facilitator of online communication. Yet, current interfaces do not offer much insight into the structure and dynamics of one's social network [12], especially with regard to communication patterns $[9,10,11]$. According to [10], users "display typical patterns of response behaviors" in email communication, and these patterns are closely related to relationships. Commonly used email interfaces present messages as visually monotonous and increasingly long lists that tend to hinder the user's ability to monitor her contacts', and maintain her own "responsiveness image" [10].

The thousands of messages in one's inbox are more than just a large collection of "to-dos" and reminders, pictures and attached documents. They are a vibrant, clear record of one's past, a diary we were never aware we were keeping. The large data sets created by the use of email make email an obvious field in which to apply visualization techniques.

In recognition of email's personal and social role, we address intimacy by visualizing it chronologically. Applying notions of intimacy, and techniques of information visualization, we have constructed faMailiar, a system that facilitates seeing rhythms of social engagement in large email collections. By rhythms, we mean significant patterns over time, which can be felt. A preliminary experience-oriented user study indicates that this visualization can make users aware of rhythms within their email usage that have otherwise remained concealed. This awareness enables them to identify relationships that require more or deserve less attention.

\section{METRICS OF INTIMACY IN EMAIL}

In order to represent intimacy in an email visualization system, we need measures that quantify it. We define intimacy computationally for an email message (or set thereof) as a combination of two metrics: contact intimacy category and message intimacy weight. 
Contact intimacy category is defined by the user. It reflects how intimate the user feels about each email contact. The user can categorize each significant contact into one of 4 categories, ranging from most to least intimate. Those not categorized are assigned by default to an additional least intimate category. As relationships between people can change over time, the user can modify contact intimacy category for any contact at any time.

Message intimacy weight is a computed parameter, based on message data. Each message is procedurally assigned an intimacy weight rating between 0 and 1 , as a result of a simple information retrieval (IR) analysis of the presence of "intimate" and "anti-intimate" syntagmata. When defining the IR criteria, we considered emotional tone, context (i.e. professional vs. casual), formality of language and capitalization. Forwarded messages and those having more than one recipient are assigned decreasing intimacy weight. While our implementation here is preliminary, it seems to be useful in practice.

\section{VISUALIZING RHYTHMS OF INTIMACY}

We are visualizing intimacy metrics in a zoomable chronological grid. This enables users to perceive patterns in the development of their relationships through email over time. Our application enables the user to define, quantify, see, filter and understand intimacy within email.

Our email visualization system was developed through a human-centered, iterative design process. Initially, we considered numerous mappings of the multi-dimensional data space to the multi-dimensional visual attributes space. Based on those mappings, ten visual designs were produced with pencil and paper. Informal discussions helped us choose three designs to develop further. We created a set of interactive mockups for each of these designs. These were subsequently evaluated through ethnographic interviews with 6 email users. We used the results of the interviews, along with established information visualization principles [2], in order to design a set of visual mappings that were implemented procedurally. We also decided to ensure that the program would integrate seamlessly with Outlook.

\section{Mapping Email Intimacy Metrics}

We map contact intimacy category and message intimacy weight into each icon, as independent visual attributes. Contact intimacy category is mapped to the shape and hue attributes (Figure 1). This is consistent with Mackinlay's findings, that hue is an effective encoding in nominal perceptual tasks [2]. We use cold-warm contrast [5] in assigning hues, mapping most intimate to a warm, yellow. Progressively less intimate contact categories are mapped to red, green, and blue (Figure 1). Messages in the fifth, default category are represented by a cold, gray hue, which, by design, almost blends into the background.

Redundantly, we employ a gradation in the number of edges of the shapes that are used to represent incoming messages. Those from the most intimate contacts are

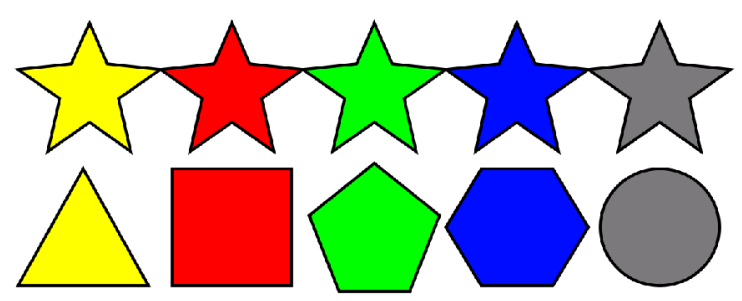

Figure 1. Outgoing (top) and incoming message shapes (bottom), ordered from left to right by decreasing contact intimacy category (yellow, red, green, blue, gray).

represented as triangles. Less intimate categories are mapped progressively with more sides (Figure 1, bottom).

Outgoing messages are mapped to a distinctly different shape (Figure 1, top). They are represented as stars. Consistent with the treatment of incoming messages, hue is assigned based on the recipient's contact intimacy category. We represent messages that the user has sent to herself (i.e. reminders) as white stars, rendering them highly visible.

Message intimacy weights are differentiated through the use of the value [5] (brightness) attribute. More intimate messages are brighter than less intimate ones. In order to facilitate perception of different message intimacy weights, the continuous numeric intimacy weight range of 0 to 1 is mapped to four discrete value levels $(.25, .5, .75$ or 1$)$.

\section{Chronological Calendar Grid}

A rhythm is a pattern over time, which has visceral impact. In order to make communication rhythms visible, we present iconographic representations of email messages in a chronological calendar grid. Since position is the most effective encoding for ordinal data [2], we use $\mathrm{x}$ and $\mathrm{y}$ position to structure a chronological grid. 2 levels of chronological scale can be chosen: daily and weekly views.

Daily view (Figure 2) is more detailed of the two. Days are shown along the $\mathrm{x}$-axis, and hours along the $\mathrm{y}$-axis. Each icon represents a message. Within each hour, incoming and outgoing messages are adjacent.

In weekly view, (Figure 3), weeks are along the X-axis, with days along y. Each icon aggregates the set of messages within a contact intimacy category for one day. The icon's size is represented proportionally to the number of messages it represents.

\section{Making Communication Rhythms Visible}

Temporal navigation enables the user to see different chronological perspectives. This helps make communication patterns over time visible, and thus facilitates perception of rhythms. One technique is to enable the user to vary the amount of data and level of detail presented. We used the Piccolo toolkit [7] to build a zoomable user interface that lets the user expand and contract the chronological range of message icons shown (Figure 4). The user can fill the screen with as little as one day, or as much as four months. Panning is also afforded, either by dragging the background or by using the arrows to activate animated scrolling at a 


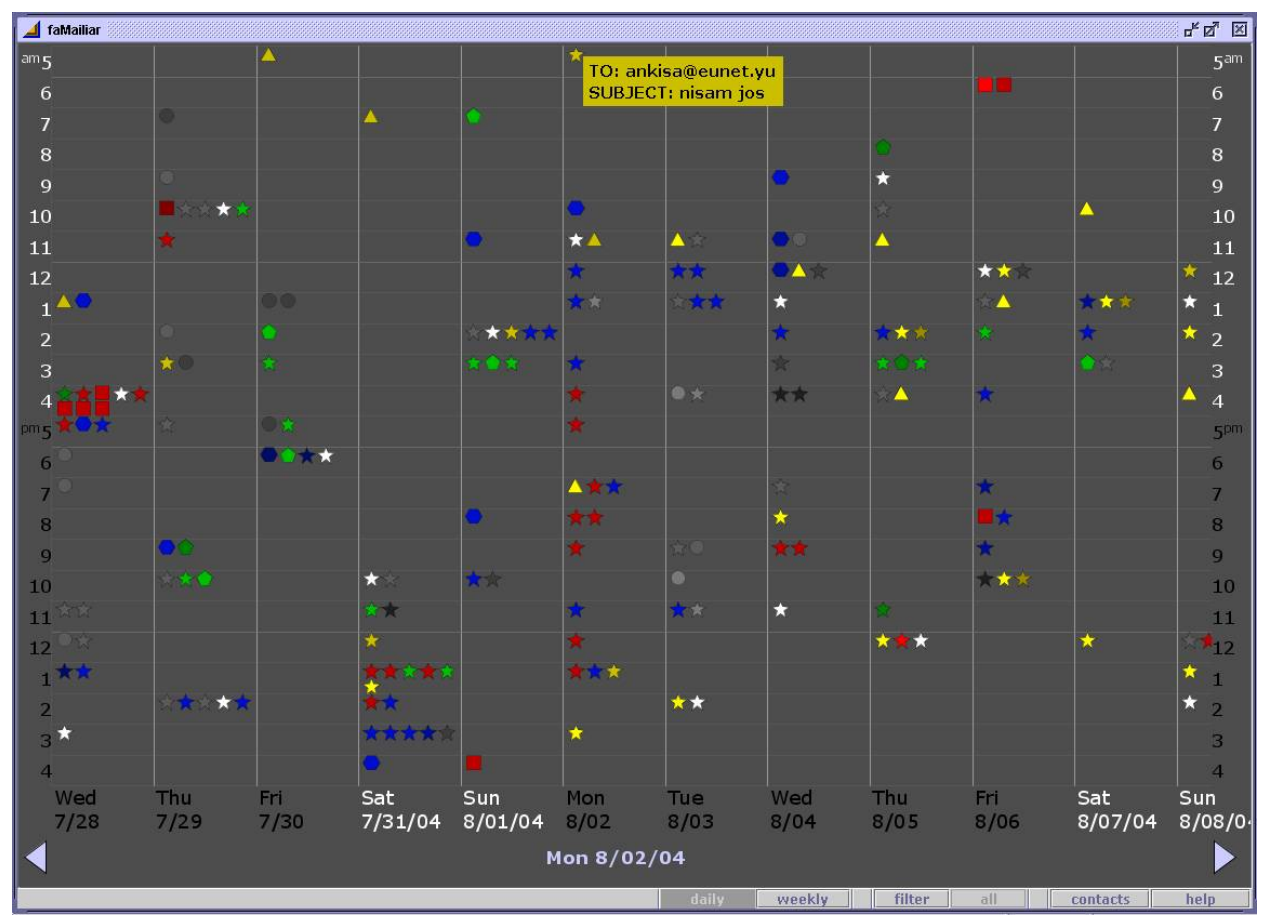

Figure 2. Daily view of a user's email. Each icon represents one message. The brief view of details on demand is shown.

speed proportional to the zoom level.

Filters permit the user to form queries to visualize a subset of the message collection. Queries can be specified via form fields (subject, body, sender and/or recipient). Or, when a message is selected, queries can be specified by example, such as by contact or message thread. Following a query, only the icons that satisfy the query are colored, while all the others are shown as silhouettes. Filtering enables the user to focus her attention on a significant subset of her email messages (Figure 4). Filtered data sets make rhythms, such as 'person A always emails me after 10 p.m.' or 'person B never responds during the weekend' visible.

Details are available on-demand [2]. In daily mode, when the user is interested in a particular message, s/he can access details at one of the three messages levels: brief

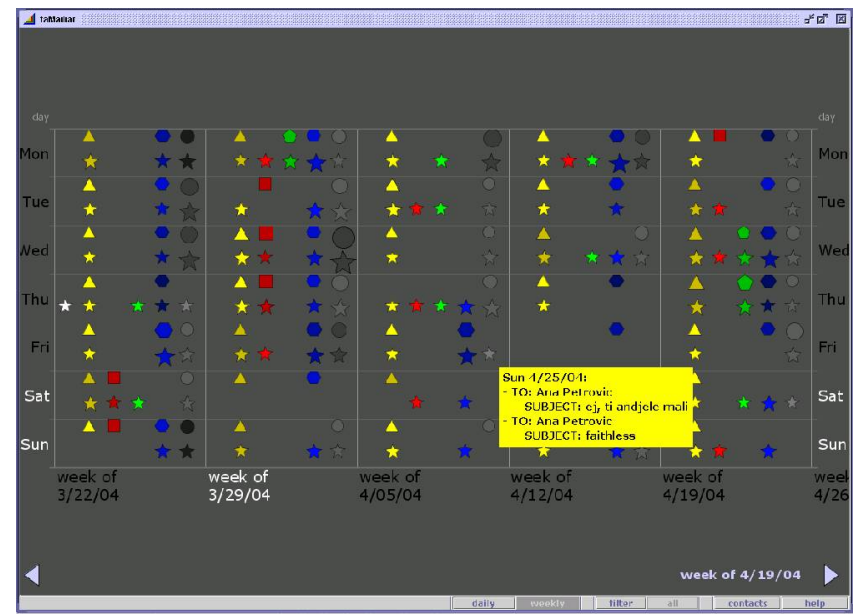

Figure 3. Weekly view of a user's email. Each icon aggregates messages for a day \& contact intimacy category. The partial view of details on demand is shown.
(Figure 2), partial, or complete. Weekly mode offers similar message group detail levels: brief and partial (Figure 3).

\section{PRELIMINARY EXPERIENCE-ORIENTED USER STUDY}

We conducted a preliminary experience-oriented user study. By nature, email is personal and private. Therefore, instead of bringing subjects into a usability lab, we gave them our software, and asked them to use it in the context of their own space. There were no specific tasks, only a general directive: to use the program as part of their processes of reading and writing email. We wanted to see how an email visualization system which uses intimacy and chronology would affect their experience.

\section{Participants and Protocol}

The preliminary study involved 6 participants. All of the participants were experienced users of Microsoft Outlook. On the average, they spent around 1.5 hours daily engaged

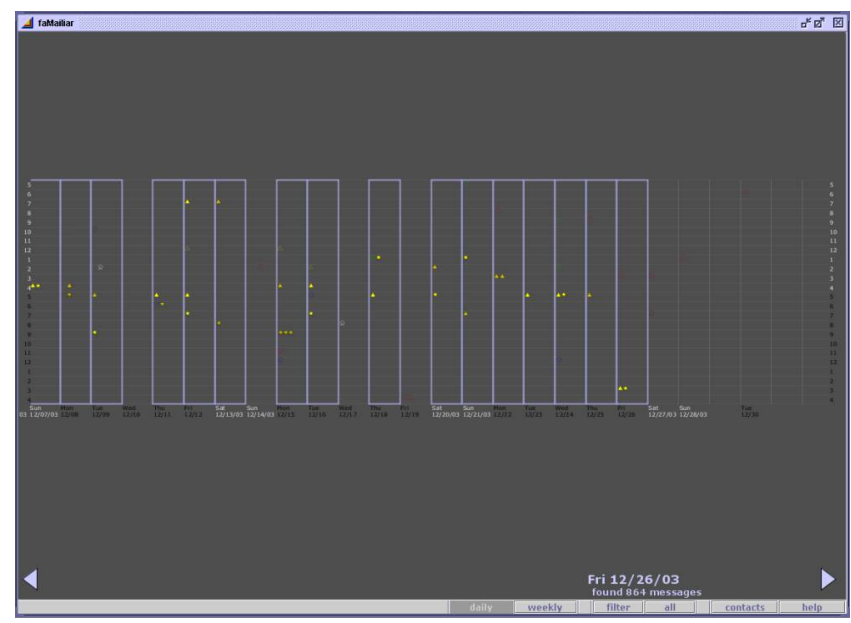

Figure 4. Zoomed out and filtered daily email view. 
in the process of sending and receiving email. The study was voluntary, with no compensation for the participants.

The study participants were instructed to use faMailiar, alongside of Outlook, at their own discretion, during a period of seven days. At the end of the week, an interview session was conducted with each participant. They reported using the intimacy visualization interface several times during each day, in alternation with the regular email client. They spent an average of 20 minutes daily with the application. The number of visualized messages was between 100 and 1,000 (average 550), covering time intervals between 3 weeks and 4 years (average 1.5 years).

\section{Results and Implications}

The most interesting results were experiential answers to the question, "Did using faMailiar enable you to learn something new about your email history?" For example, one of the participants noted, "I could see that in past four years I lost many of my personal relationships in favor of school." Another participant was surprised by the amount of email he wrote and concluded that his sleep patterns were not healthy, based on the erratic distribution of the messages he sent. Yet another participant observed that "it was depressing that nobody emailed [her] after midnight." Several participants pointed out that just by observing the patterns in the visualization, they were able to quickly remember past activities and contacts involved.

Psychological research indicates that experiences of intimacy and connectedness through social networks are vital to human sense of well-being [6,8]. Participants' responses demonstrate that through the experience of intimacy-based email visualization, they gained fundamental new insights into their lives. These insights potentially enable users to better manage how they invest time and energy into personal and work relationships, and thus to improve overall sense of well-being. To continue this process, all of the participants asked to keep the software and continue using it after the study

While we have not yet measured statistical significance, the quantitative study data is consistent. For example, compared to the email interfaces they used regularly, the participants felt that they could predict with more accuracy the exact time of the day/month that any specific contact would next email them (4.25/5 on the likert-scale), as well as exactly how long it would take a specific contact to respond after receiving an email from them (4/5).

\section{CONCLUSIONS}

In the barrage of daily email messages, people easily lose track of their communication experiences over time, and the statuses of relationships that are involved. Information visualization is a means for making patterns in large data sets visible. We used chronology and two computational measures of intimacy as the basis for applying information visualization techniques to personal email collections. Our goals were to provide a more personal experience of the user's electronic habitat, and give users a new perspective on the evolution of the intimate dimensions of their communication. Our hypothesis was that this approach would provide insight into the rhythms of relationships and personal experiences that flow through the medium of email.

The preliminary experience study showed that a system that uses intimacy and chronology to visualize rhythms in email experience without disrupting their usual email practices enables users to understand their email collections in new, meaningful ways. They can see some previously hidden, important facets of how they conduct their personal relationships. Intimate relationships are essential to humans' sense of well-being [8]. Thus, seeing rhythms of intimacy in email gives users the opportunity to assign personal attention to intimate relationships, and thus to improve their overall sense of well-being.

\section{REFERENCES}

1. Bellotti, V., Ducheneaut, N., Howard, M., and Smith, I. Taking email to task: design and eval of a task management centered email tool. Proc. CHI 2003, 345-352.

2. Card, S., Mackinlay, J., and Shneiderman, B. Readings in Information Visualization: Using Vision to Think. Morgan Kaufmann, San Francisco, CA, 1999.

3. Gross, B. Navigation, organization and retrieval in personal collections of email. Proc. CSCW 2002 Workshop: Redesigning Email for the $21^{\text {st }}$ Century.

4. Gwizdka, J. TaskView - design and evaluation of a taskbased email interface. Proc. 2002 Conf of the Centre for Advanced Studies on Collaborative Research, 4-14.

5. Itten, J. The art of color. Van Nostrand Reinhold, New York, NY, 1973

6. Jamieson, L. Intimacy: personal relationships in modern societies. Polity Press, Cambridge, MA, 1998.

7. Piccolo. http://www.cs.umd.edu/hcil/piccolo/.

8. Prager, K.J. The psychology of intimacy. Guilford, New York, NY, 1995.

9. Rohall, S., L., Gruen, D., Moody, P., and Kellerman, S. 2001. Email visualizations to aid communications. Proc. IEEE InfoVis 2001, 12-15.

10. Tyler, J., and Tang, J. When can I expect an email response? A study of rhythms in email usage. Proc. ECSCW 2003.

11. Venolia, G., and Neustaedter, C. Understanding sequence and reply relationships within email conversations: a mixed-model visualization. Proc. CHI 2003, 361-368.

12. Viegas, F., boyd, d., Nguyen, D, Potter, J., and Donath, J. Digital artifacts for remembering and storytelling: PostHistory and Social Network Fragments. Proc. HICSS-37. http://csdl.computer.org/comp/proceedings /hicss/2004 /2056/04/205640109aabs.htm, 2004.

13. Whittaker, S., and Sidner, C. Email overload: exploring personal info management of email. Proc. CHI 1996. 